\title{
Baryon-antibaryon dynamics in relativistic heavy-ion collisions
}

\author{
E. Seifert and W. Cassing \\ Institut für Theoretische Physik, Universität Gießen, D-35392 Giessen, Germany
}

(Received 25 January 2018; published 16 April 2018)

\begin{abstract}
The dynamics of baryon-antibaryon annihilation and reproduction $(B \bar{B} \leftrightarrow 3 M)$ is studied within the PartonHadron-String Dynamics (PHSD) transport approach for $\mathrm{Pb}+\mathrm{Pb}$ and $\mathrm{Au}+\mathrm{Au}$ collisions as a function of centrality from lower Super Proton Synchrotron (SPS) up to Large Hadron Collider (LHC) energies on the basis of the quark rearrangement model. At Relativistic Heavy-Ion Collider (RHIC) energies we find a small net reduction of baryon-antibaryon $(B \bar{B})$ pairs while for the LHC energy of $\sqrt{s_{N N}}=2.76 \mathrm{TeV}$ a small net enhancement is found relative to calculations without annihilation (and reproduction) channels. Accordingly, the sizable difference between data and statistical calculations in $\mathrm{Pb}+\mathrm{Pb}$ collisions at $\sqrt{s_{N N}}=2.76 \mathrm{TeV}$ for proton and antiproton yields [ALICE Collaboration, B. Abelev et al., Phys. Rev. C 88, 044910 (2013)], where a deviation of $2.7 \sigma$ was claimed by the ALICE Collaboration, should not be attributed to a net antiproton annihilation. This is in line with the observation that no substantial deviation between the data and statistical hadronization model (SHM) calculations is seen for antihyperons, since according to the PHSD analysis the antihyperons should be modified by the same amount as antiprotons. As the PHSD results for particle ratios are in line with the ALICE data (within error bars) this might point towards a deviation from statistical equilibrium in the hadronization (at least for protons and antiprotons). Furthermore, we find that the $B \bar{B} \leftrightarrow 3 M$ reactions are more effective at lower SPS energies where a net suppression for antiprotons and antihyperons up to a factor of 2-2.5 can be extracted from the PHSD calculations for central $\mathrm{Au}+\mathrm{Au}$ collisions.
\end{abstract}

DOI: 10.1103/PhysRevC.97.044907

\section{INTRODUCTION}

Relativistic and ultrarelativistic heavy-ion collisions offer the unique possibility to study a new phase of matter, i.e., a quark-gluon plasma (QGP), as well as possibly the phase boundary between the hadronic and partonic phase. Lattice quantum chromodynamics (1QCD) calculations suggest that at vanishing baryon chemical potential $\left(\mu_{B}=0\right)$ there is a crossover phase transition from hadronic to partonic degrees of freedom [1-6] for the deconfinement phase transition as well as for the restoration of chiral symmetry. However, at some finite baryon chemical potential the crossover might turn to a first-order phase transition implying a critical endpoint in the QCD phase diagram [7]. Since lattice calculations so far suffer from the fermion-sign problem, no first principle information on the phase boundary can be extracted from 1QCD at large $\mu_{B}$, whereas at low $\mu_{B}$ Taylor expansions of the thermodynamic potential (in powers of $\mu_{B} / T$ ) provide an alternative solution. The studies in Refs. [8,9] show that for heavy-ion reactions at Relativistic Heavy-Ion Collider (RHIC) and Large Hadron Collider (LHC) energies the phase boundary is a crossover and the critical temperature for deconfinement $T_{c}$ is practically the same as at $\mu_{B}=0$.

Published by the American Physical Society under the terms of the Creative Commons Attribution 4.0 International license. Further distribution of this work must maintain attribution to the author(s) and the published article's title, journal citation, and DOI. Funded by $S C O A P^{3}$.
Due to the high energy densities reached in $\mathrm{Au}+\mathrm{Au}$ $(\mathrm{Pb}+\mathrm{Pb})$ collisions at RHIC and LHC energies as well as strong partonic interactions the final hadron yields turn out experimentally to be close to thermal and chemical equilibrium as described by a grand-canonical ensemble of noninteracting hadronic states (with excluded volume corrections) [10-17]. In fact, the thermal analysis of hadron yields at midrapidity show a high degree of thermalization [18]; however, a sizable difference between data and statistical calculations pop up in $\mathrm{Pb}+\mathrm{Pb}$ collisions at $\sqrt{s_{N N}}=2.76 \mathrm{TeV}$ for proton and antiproton yields [19], where a deviation of $2.7 \sigma$ is obtained [18]. It has been argued in Refs. $[19,20]$ that this deviation might be due to final-state hadronic $B \bar{B}$ annihilation after chemical freezeout. On the other hand such a reduction was not seen in the relative yields of strange baryons and antibaryons to pions [21]. In Refs. [20] the Ultrarelativistic Quantum Molecular Dynamics (UrQMD) transport model [22,23] has been employed as an hadronic "afterburner" to evaluate the final-state interactions and in particular the effects from $B \bar{B}$ annihilation after chemical freezeout; however, the backward channels had been discarded thus violating detailed balance [24]. This issue has been further addressed in Ref. [25] in a simplified model for the space-time evolution but incorporating detailed balance for the chemical reactions. In the latter study it was found that a net $B \bar{B}$ reduction by annihilation in central $\mathrm{Pb}+\mathrm{Pb}$ collisions at $\sqrt{s_{N N}}=2.76 \mathrm{GeV}$ of $\sim 40 \%$ might result thus coping approximately with the experimental observation in Ref. [19]. A more refined approach-incorporating detailed balance-has been proposed in Ref. [26] which solves chemical rate equations on top of $2+1$ hydrodynamic evolution. 
At RHIC energies the authors report a reduction of $B \bar{B}$ pairs by about $15-20 \%$; results for LHC energies from this model are not known to the authors. Nevertheless, the impact of $B \bar{B}$ annihilation and reproduction by the inverse many-body channels should be calculated on a fully microscopic basis including detailed balance.

A first step in this direction has been taken in Ref. [27] where the three-body fusion of nonstrange pseudoscalar and vector mesons to $B \bar{B}$ pairs has been incorporated in the Hadron-String Dynamics (HSD) transport approach [28] that preferentially describes the hadronic phase and provides results close to the UrQMD transport model [22,23] for Super Proton Synchrotron (SPS) energies as demonstrated in Refs. [29,30]. In Ref. [27] the matrix element squared for baryon-antibaryon annihilation has been extracted from the experimental data on $p \bar{p}$ annihilation and the three-body meson channels have been determined on the basis of detailed balance. It was found that in central collisions of heavy nuclei at SPS energies the annihilation of antinucleons is almost compensated by the inverse recreation channels. A recent extension of the model has been presented in Ref. [31] within the Parton-HadronString Dynamics (PHSD) approach [32-34] where the full strangeness sector has been included for the $2 \leftrightarrow 3$ reactions. The resulting model (denoted by PHSD4.0) has been applied to central $\mathrm{Pb}+\mathrm{Pb}$ collisions in the SPS energy regime and it was found again that $B \bar{B}$ annihilation and reproduction compensate each other to a large extent.

We recall that the PHSD transport approach [32-34] superseeds the HSD approach by a couple of aspects that become essential with increasing bombarding energy:

(i) the formation of an initial partonic phase with quark and gluon quasiparticle properties that are fitted to lattice QCD results in thermodynamic equilibrium,

(ii) a dynamical hadronization scheme on the basis of covariant transition rates,

(iii) inclusion of further hadronic reactions in the strangeness sector with full baryon-antibaryon symmetry, and

(iv) inclusion of essential aspects of chiral symmetry restoration in the hadronic phase [35].

Whereas the latter developments are important for the lower SPS energy regime to account for the strangeness enhancement seen experimentally in heavy-ion collisions, the formation of a partonic phase is mandatory to understand the physics at higher SPS, RHIC, and LHC energies. This has been demonstrated in a couple of PHSD studies in the past for heavy-ion reactions from $\sqrt{s_{N N}}=4 \mathrm{GeV}$ to $2.76 \mathrm{TeV}$ [36-39]. Since multistrange baryons and antibaryons at top SPS energies and above no longer stem from string fragmentation (as in HSD [27]) but preferentially from hadronization at energy densities around $0.5 \mathrm{GeV} / \mathrm{fm}^{3}$ the issue of three-meson fusion reactions for the formation of baryon-antibaryon $(B \bar{B})$ pairs and the annihilation of $B \bar{B}$ pairs to multiple mesons has to be investigated (in addition to Ref. [31]) at RHIC and LHC energies.

This work is organized as follows: In Sec. II we recapitulate shortly the ingredients of PHSD and the quark rearrange- ment model for baryon-antibaryon annihilation and recreation $(B \bar{B} \leftrightarrow 3 M)$ in the version 4.0 [31]. In Sec. III we present results for antibaryons and multistrange baryons from PHSD simulations for central $\mathrm{Pb}+\mathrm{Pb}(\mathrm{Au}+\mathrm{Au})$ collisions at $\mathrm{RHIC}$ and LHC energies in comparison to experimental data and then focus on the centrality dependence of baryons and antibaryons. We will compare simulations using (i) the baryon-antibaryon annihilation and formation, (ii) only $(B \bar{B})$ annihilation, and (iii) without the $2 \leftrightarrow 3$ channels.

Global excitation functions for mesons, baryons, and antibaryons will be provided in Sec. IV as well as excitation functions for the impact of final-state interactions and in particular the $B \bar{B} \leftrightarrow 3 M$ reactions. We conclude our study with a summary in Sec. V.

\section{REMINDER OF THE PHSD TRANSPORT APPROACH}

The PHSD is a microscopic covariant transport approach whose formulation is based on the Kadanoff-Baym equations [40-43] for Green's functions in phase-space representation in first-order gradient expansion beyond the quasiparticle approximation [44]. The PHSD transport approach describes in a consistent manner the whole time evolution of a relativistic heavy-ion collision as it incorporates a hadronic and a partonic phase as well as dynamical transitions between the respective degrees of freedom. The properties of the quarks, antiquarks and gluons in the QGP phase are described by the dynamical quasiparticle model (DQPM) [45,46], whose three parameters are fixed to reproduce the 1QCD equation of state at vanishing baryon chemical potential and which is based on effective propagators for the partons. The quarks and gluons have finite masses as well as widths that are given, respectively, by the real and imaginary parts of the retarded self-energies resulting from two-particle-irreducible diagrams of the effective full propagators.

In PHSD simulations of nucleus-nucleus collisions color neutral strings (described by the FRITIOF Lund model [47]) are formed from the initial hard nucleon-nucleon scatterings. These strings decay into "prehadrons" with a formation time of $\approx 0.8 \mathrm{fm} / c$ in which they do not interact. The string ends are identified with "leading hadrons" and may interact instantly with reduced cross sections according to the constituent quark model [28]. In case that the local energy density surpasses the critical value of $\epsilon_{c} \approx 0.5 \mathrm{GeV} / \mathrm{fm}^{3}$ the prehadrons dissolve into colored effective quarks, antiquarks, and gluons given by the DQPM at the given local energy density. These partons then propagate in their self-generated mean field and interact via quasielastic $2 \rightarrow 2$ collisions between quarks, antiquarks, and gluons. Additionally, $q \bar{q}$ pairs may annihilate into a gluon and gluons may decay to $q \bar{q}$ pairs. As the system expands the local energy density will drop close to or below $\epsilon_{c}$ and will start to hadronize into off-shell mesons and baryons. During hadronization the energy, three-momentum, and quantum numbers are conserved in each event [32]. In the hadronic phase the particles interact with each other (as in HSD) via elastic and inelastic collisions satisfying the detailed balance relations. The cross sections are taken from experiments or effective models. 


\section{A. Quark rearrangement model for $B+\bar{B}$ production and annihilation}

The quark rearrangement model (in the context of $B \bar{B}$ annihilation and reproduction) goes back to Ref. [27] and has been extended to the $\mathrm{SU}(3)_{\text {flavor }}$ sector recently [31]. It is based on the experimental observation of a dominant annihilation of $p \bar{p}$ into (on average) five pions at invariant energies $2.3 \mathrm{GeV} \leqslant$ $\sqrt{s} \leqslant 4 \mathrm{GeV}$. Now the final number of five pions may be interpreted as an initial annihilation into $\pi \rho \rho$ with the $\rho$ mesons decaying subsequently into two pions each. The channel $\pi \pi \rho$ then leads to four final pions, the channel $\pi \omega \rho$ to six final pions, the channel $\rho \omega \rho$ to seven final pions, etc. Accordingly, the baryon-antibaryon annihilation in the first step is a $2 \rightarrow 3$ reaction with a conserved number of quarks and antiquarks. This is the basic assumption of the quark rearrangement model which is illustrated in Fig. 2 of Ref. [31]. By allowing the mesons $M_{i}$ to be any member of the $0^{-}$or $1^{-}$nonets one can describe an arbitrary $B \bar{B}$ annihilation and recreation by rearranging the quark and antiquark content, where $B$ is a member of the baryon octet or decuplet. This approach gives a realistic description for $p \bar{p}$ annihilation and we assume that for other baryon-antibaryon pairs than $p \bar{p}$ a similar annihilation pattern holds. Since there are no measurements of annihilation cross sections other than $n \bar{p}$ and $p \bar{p}$ this is our best guess at present.

\section{B. $2 \leftrightarrow 3$ reactions in kinetic theory}

The treatment of $2 \leftrightarrow 3$ reactions can be incorporated in the collision term in kinetic theory on a channel-by-channel basis by employing detailed balance as formulated in Refs. [27,31]. The matrix elements squared $\left|M_{B \bar{B}}^{c}(\sqrt{s})\right|^{2}$ for a channel $c$, which - apart from phase-space integrals—determine the $B \bar{B}$ annihilation rate, are assumed to be the same for all flavor channels $c$. We recall that more than 2500 individual mass channels are incorporated and the three-body phase-space integrals have to be evaluated for each of these channels as a function of invariant energy $\sqrt{s}$ [31]. The technical solution to this problem and detailed tests of the algorithm in a finite box with periodic boundary conditions have been described in Ref. [31]. The matrix element squared $\left|M_{B \bar{B}}(\sqrt{s})\right|^{2}$ has been extracted from the experimental data on $p \bar{p}$ annihilation as a function of the relative momentum and it has been assumed that the product of relative velocity (in the center-of-mass system) $v_{\text {rel }}$ and annihilation cross section $\sigma_{\text {ann }}$ for other flavor channels is the same as for the channel $p \bar{p}$. In this work we discard an explicit strangeness suppression factor which was found in Ref. [31] (Fig. 19) to have a minor impact on the actual results for relativistic heavy-ion collisions. Within these assumptions the kinetic approach has no additional free parameter when incorporating the $2 \leftrightarrow 3$ reactions in addition to the $1 \leftrightarrow 2$ and $2 \leftrightarrow 2$ channels [36]. The actual solution of the covariant transition rates in case of heavy-ion collisions is performed by Monte Carlo employing the in-cell method as in Refs. [27,31].

\section{PHSD SIMULATIONS FOR HEAVY-ION COLLISIONS}

In this section we show the influence of the $B \bar{B} \leftrightarrow 3 M$ reactions on heavy-ion collisions in particular at RHIC and LHC energies in extension of the calculations at SPS energies

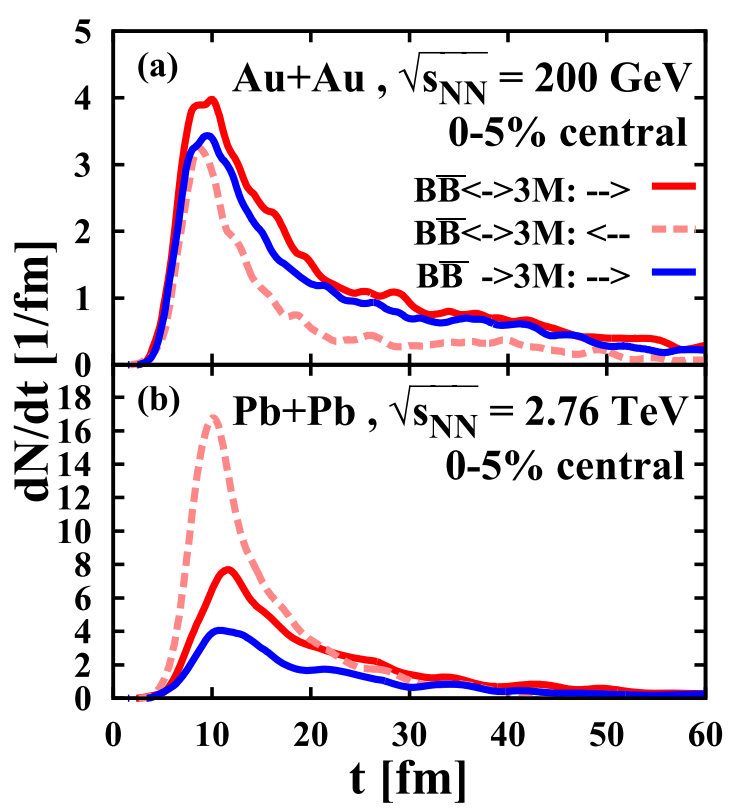

FIG. 1. The reaction rates of the $B \bar{B} \rightarrow 3 M$ reactions (solid line) as a function of time in $5 \%$ central $\mathrm{Au}+\mathrm{Au}$ collisions at $\sqrt{s_{N N}}=$ $200 \mathrm{GeV}$ (a) and $\mathrm{Pb}+\mathrm{Pb}$ collisions at $\sqrt{s_{N N}}=2.76 \mathrm{TeV}$ (b) integrated over rapidity. The solid blue lines denote the rates for $B \bar{B}$ annihilation when discarding the reproduction channels; the red solid lines stand for the $B \bar{B}$ annihilation rate when including the backward channels whereas the dashed lines display the reproduction rate in the latter case.

in Ref. [31]. Before coming to the actual results for hadron spectra we compare in Fig. 1 the reaction rates for the total baryon-antibaryon annihilation and formation from PHSD in $5 \%$ central $\mathrm{Pb}+\mathrm{Pb}(\mathrm{Au}+\mathrm{Au})$ collisions at $\sqrt{s_{N N}}=200 \mathrm{GeV}$ (a) and $2.76 \mathrm{TeV}$ (b) integrated over rapidity. The solid blue lines denote the rates for $B \bar{B}$ annihilation when discarding the reproduction channels; the red solid lines stand for the $B \bar{B}$ annihilation rate when including the backward channels, whereas the dashed lines display the reproduction rate in the latter case. The meson-fusion rate dominates at early times at the LHC energy over the $B \bar{B}$ annihilation rate (b) while the situation is inverse at the top RHIC energy (a). Without regeneration of $B \bar{B}$ pairs (blue solid lines) the annihilation rates are lower than in case of $B \bar{B}$ reproduction which is, however, an unphysical limit and displayed only for orientation. The explicit dependence of ratios versus $\sqrt{s_{N N}}$ will be discussed in Sec. IV.

\section{A. Hadron transverse mass spectra at RHIC and LHC energies}

We recall that rapidity and transverse-momentum spectra of antibaryons from PHSD in central $\mathrm{Pb}+\mathrm{Pb}$ collisions at SPS energies have been shown in Ref. [31] in comparison to the available data. We here continue with PHSD results for antibaryons and mesons in 5\% central $\mathrm{Pb}+\mathrm{Pb}(\mathrm{Au}+\mathrm{Au})$ collisions at the top RHIC energy $\left(\sqrt{s_{N N}}=200 \mathrm{GeV}\right)$ and the LHC energy of $\sqrt{s_{N N}}=2.76 \mathrm{TeV}$. In Fig. 2 we display the calculated transverse-momentum spectra for protons and positive and negative pions as well as for kaons and antikaons in comparison to the data from the PHENIX Collaboration [48]. Whereas the hadron spectra are quite well described at lower 


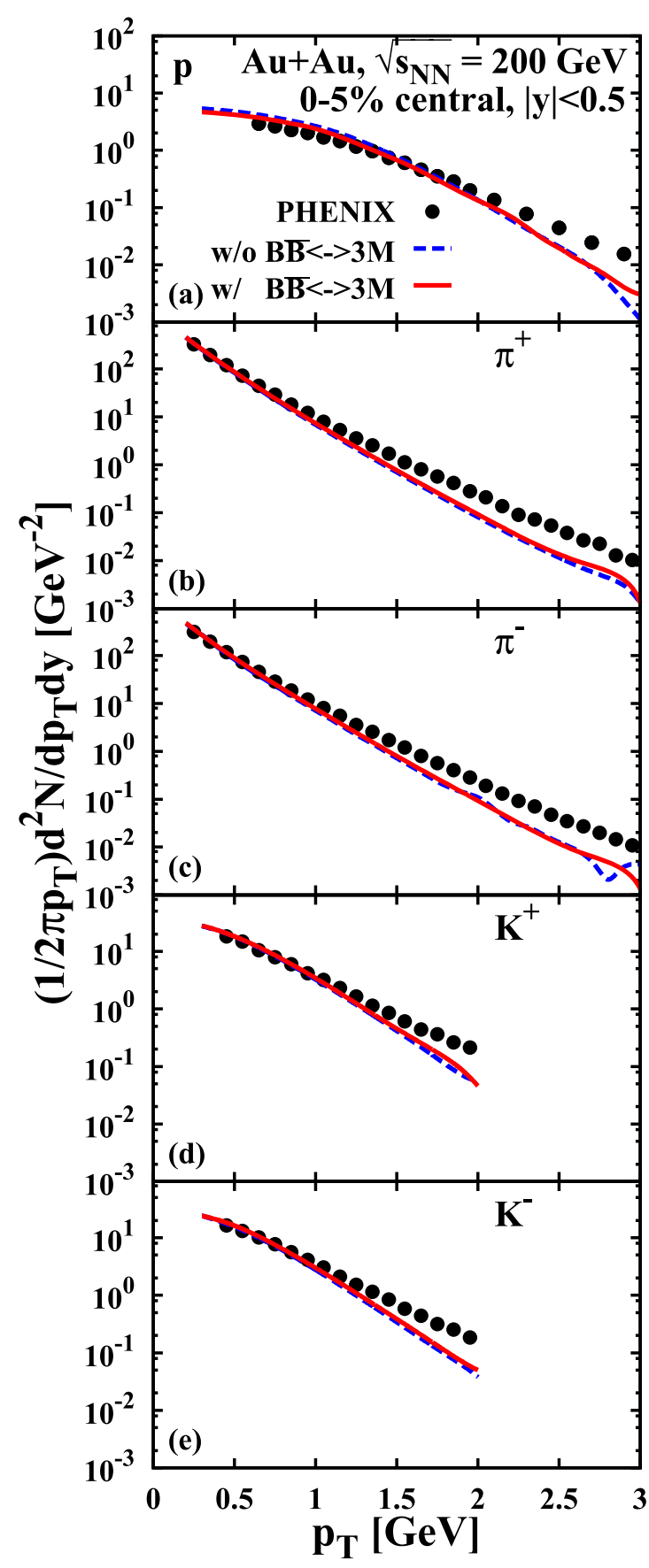

FIG. 2. The transverse-momentum spectra for protons and positive and negative pions as well as for kaons and antikaons from PHSD at midrapidity in comparison to the data from the PHENIX Collaboration [48] for 5\% central $\mathrm{Au}+\mathrm{Au}$ collisions at $\sqrt{s_{N N}}=200 \mathrm{GeV}$. The full red lines show the results of calculations with the $2 \leftrightarrow 3$ reactions included while dashed lines correspond to calculations with the $2 \leftrightarrow 3$ reactions discarded.

transverse momenta, there is a deficit at high $p_{T}$ for all hadron species in the PHSD calculations. We note that the hadron formation at the top RHIC energy at midrapidity proceeds essentially by hadronization, i.e., by dynamical coalescence, which implies that the quarks and antiquarks at hadronization have softer transverse momenta in PHSD than in "experiment."
The total hadron densities at midrapidity are only marginally affected by the underestimated high $p_{T}$ tail and we may conclude that the hadron densities within PHSD are sufficiently realistic such that rather solid results for the annihilation and fusion rates should emerge. The full red lines show the spectra from calculations with the $2 \leftrightarrow 3$ reactions included while the dashed lines correspond to calculations with the $2 \leftrightarrow 3$ reactions discarded. Since there are almost no differences between the lines we can conclude again that the $2 \leftrightarrow 3$ reactions have practically no impact on baryon and meson spectra (cf. Ref. [31] for SPS energies).

In Fig. 3 we show the same hadron $p_{T}$ spectra at midrapidity for $5 \%$ central $\mathrm{Pb}+\mathrm{Pb}$ collisions at $\sqrt{s_{N N}}=2.76 \mathrm{TeV}$ in comparison to the data from the ALICE Collaboration [49-52]. In this case the description of the data is rather good (except for protons) and again there is no visible impact of the $2 \leftrightarrow 3$ reactions on these transverse-momentum spectra. We note in passing that the flow coefficients $v_{n}$ (for $n=2,3,4,5$ ) from PHSD for this system are also in a very good agreement with the experimental measurements as shown in Ref. [53]. Thus we may state that the densities of the most abundant hadrons appear to be well under control in PHSD in particular at the LHC energy.

We continue with the antibaryon transverse-momentum spectra at midrapidity for top RHIC and LHC energies, which are displayed in Figs. 4 and 5, respectively, in comparison with the data from the PHENIX, STAR, and ALICE Collaborations $[19,48,50,51,54,55]$. Here again the low-momentum spectra for $\bar{p}, \bar{\Lambda}+\bar{\Sigma}^{0}, \Xi^{-}, \bar{\Xi}^{+}$, and $\Omega^{-}+\bar{\Omega}^{+}$are roughly described at low momenta; however, the high $p_{T}$ tails are missed considerably at $\sqrt{s_{N N}}=200 \mathrm{GeV}$ in Fig. 4 while they look somewhat better at the LHC energy in Fig. 5. We note that again there is no visible impact of the $2 \leftrightarrow 3$ reactions on these transverse-momentum spectra.

\section{B. Time evolution of the antiproton and proton yield at midrapidity in different scenarios}

Some further information on the role of the $2 \leftrightarrow 3$ reactions can be extracted from the actual time evolution of the baryon and antibaryon yields. In Fig. 6 we display the number of formed protons and antiprotons (for $|y|<0.5$ ) as a function of time for a central $\mathrm{Pb}+\mathrm{Pb}$ collision at $\sqrt{s_{N N}}=2.76 \mathrm{TeV}$ in the following scenarios:

(i) HSD calculation without any annihilation and recreation channels $(B \bar{B} \leftrightarrow 3 M)$,

(ii) HSD calculation with only the annihilation $(B \bar{B} \rightarrow$ $3 M$ ) channels,

(iii) HSD calculation with all $B \bar{B} \leftrightarrow 3 M$ channels included,

(iv) PHSD calculation without the $B \bar{B} \leftrightarrow 3 M$ channels,

(v) PHSD calculation with only the $B \bar{B} \rightarrow 3 M$ channels,

(vi) PHSD calculation with all $B \bar{B} \leftrightarrow 3 M$ channels included.

Whereas the first three scenarios do not incorporate any partonic phase, the last three scenarios do such that a direct comparison allows to study the relative impact of the QGP phase and the role of the $2 \leftrightarrow 3$ reactions. We find that the time 


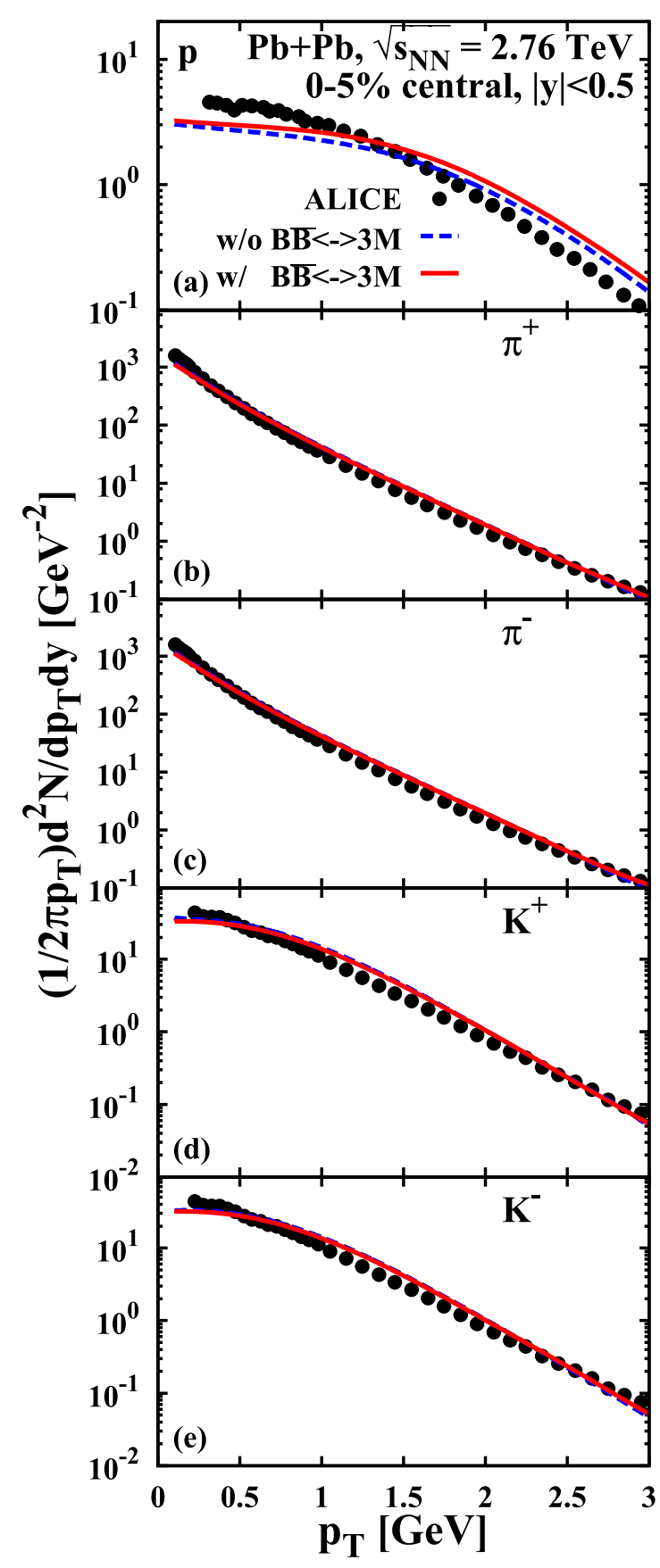

FIG. 3. The transverse-momentum spectra for protons and positive and negative pions as well as for kaons and antikaons from PHSD at midrapidity in comparison to the data from the ALICE Collaboration $[19,49-52]$ for $5 \%$ central $\mathrm{Pb}+\mathrm{Pb}$ collisions at $\sqrt{s_{N N}}=2.76$ $\mathrm{TeV}$. The full red lines show the results of calculations with the $2 \leftrightarrow 3$ reactions included while dashed lines correspond to calculations with the $2 \leftrightarrow 3$ reactions discarded.

evolution of the protons is very similar to that of the antiprotons in all scenarios considered. This is essentially due to the fact that at this energy (and midrapidity) the baryon chemical potential is approximately zero and particle-antiparticle reactions are treated on the same footing in HSD and PHSD. We recall that in HSD "formed" hadrons only appear for energy densities of $\epsilon<0.5 \mathrm{GeV} / \mathrm{fm}^{3}$ as well as hadronic

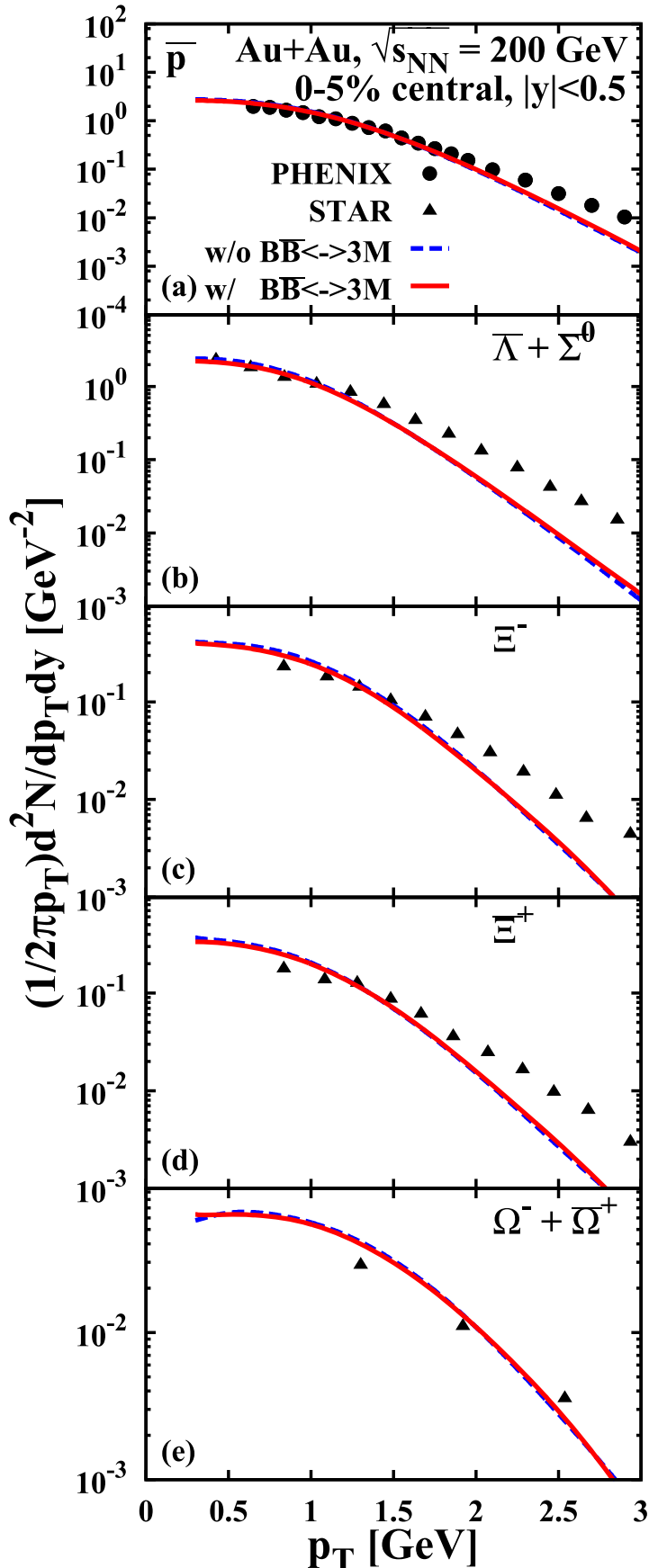

FIG. 4. The transverse-momentum spectra for $\bar{p}, \bar{\Lambda}+$ $\bar{\Sigma}^{0}, \Xi^{-}, \bar{\Xi}^{+}$, and $\Omega^{-}+\bar{\Omega}^{+}$from PHSD at midrapidity in comparison to the data from the PHENIX and STAR Collaborations $[48,54,55]$ for $5 \%$ central $\mathrm{Au}+\mathrm{Au}$ collisions at $\sqrt{s_{N N}}=200 \mathrm{GeV}$. The full red lines show the results of calculations with the $2 \leftrightarrow 3$ reactions included while dashed lines correspond to calculations with the $2 \leftrightarrow 3$ reactions discarded.

scatterings and that the production of midrapidity particles is dominated by PYTHIA6.4, whereas in PHSD (especially at $\sqrt{s_{N N}}=2.76 \mathrm{TeV}$ ) the midrapidity particles are produced by hadronization at energy densities $\epsilon \approx 0.5 \mathrm{GeV} / \mathrm{fm}^{3}$. Thus formed hadrons in both models appear at roughly the same time but their production mechanism is different and the particles 


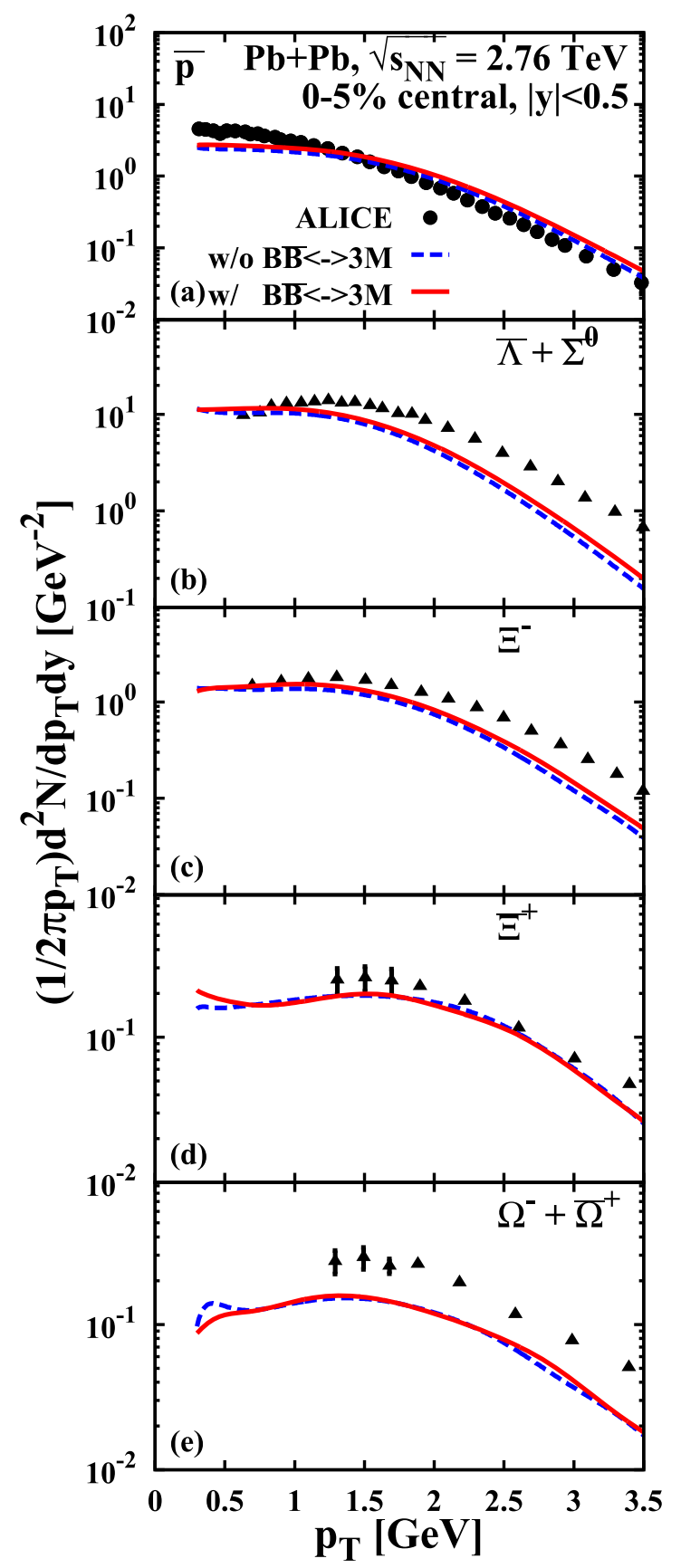

FIG. 5. The transverse-momentum spectra for $\bar{p}, \bar{\Lambda}+$ $\bar{\Sigma}^{0}, \Xi^{-}, \bar{\Xi}^{+}$, and $\Omega^{-}+\bar{\Omega}^{+}$from PHSD at midrapidity in comparison to the data from the ALICE Collaboration $[19,50,51]$ for $5 \%$ central $\mathrm{Pb}+\mathrm{Pb}$ collisions at $\sqrt{s_{N N}}=2.76 \mathrm{TeV}$. The full red lines show the results of calculations with the $2 \leftrightarrow 3$ reactions included while dashed lines correspond to calculations with the $2 \leftrightarrow 3$ reactions discarded.

from hadronization in PHSD carry the collective flow from the interacting partonic phase. In general, the yield of protons and antiprotons is higher from PYTHIA6 . 4 (in HSD) than that from hadronization (in PHSD) by almost $30 \%$ as seen from the ratio of the blue dotted lines in Fig. 6, where the $2 \leftrightarrow 3$ reactions are discarded. On the other hand, from PHSD we

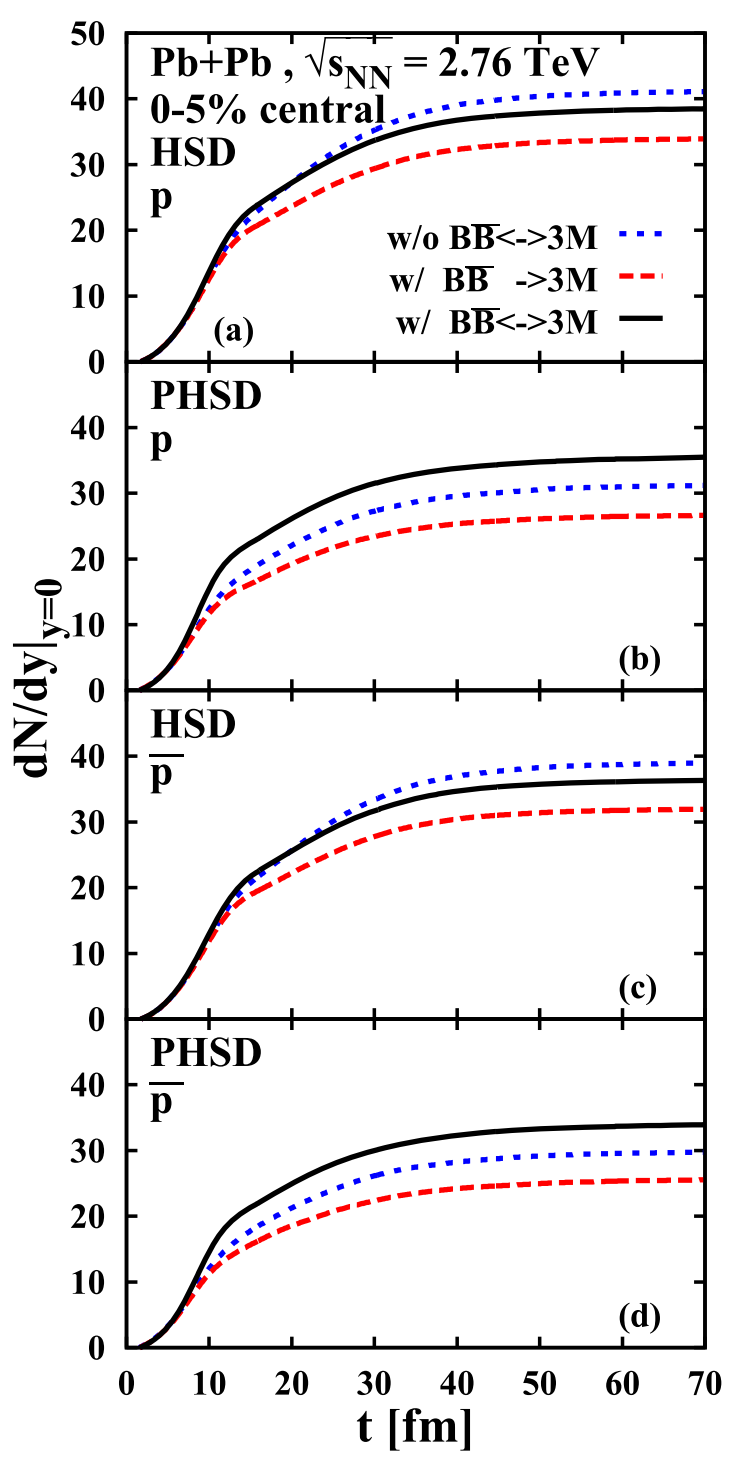

FIG. 6. Time evolution of the midrapidity yields of the protons [(a) and (b)] and antiprotons [(c) and (d)] in $0-5 \%$ central $\mathrm{Pb}+\mathrm{Pb}$ collisions at $\sqrt{s_{N N}}=2.76 \mathrm{TeV}$ from HSD [(a) and (c)] and PHSD [(b) and (d)]. The dashed red lines show the results of calculations with only $B \bar{B}$ annihilation, the solid black lines show results with the $2 \leftrightarrow 3$ reactions included while the dotted blue lines correspond to calculations with the $2 \leftrightarrow 3$ reactions discarded.

get about $10 \%$ more pions than from HSD at midrapidity in this limit such that a higher three-meson fusion rate can be expected in PHSD and a higher annihilation rate in HSD. When switching on the annihilation channels (dashed red lines) a net proton and antiproton reduction of about $20 \%$ shows up for HSD and roughly $18 \%$ for PHSD. However, when accounting for all $B \bar{B} \leftrightarrow 3 M$ channels (solid black lines) the proton and antiproton abundances are larger than those without the $2 \leftrightarrow 3$ reactions in case of PHSD (by 12\%) while for HSD the full calculations still show a tiny net annihilation (by 7\%). The final proton and antiproton midrapidity yields-with the $2 \leftrightarrow 3$ reactions included - are rather close for HSD and PHSD and differ only by $\sim 6 \%$, which demonstrates that the $2 \leftrightarrow 3$ 


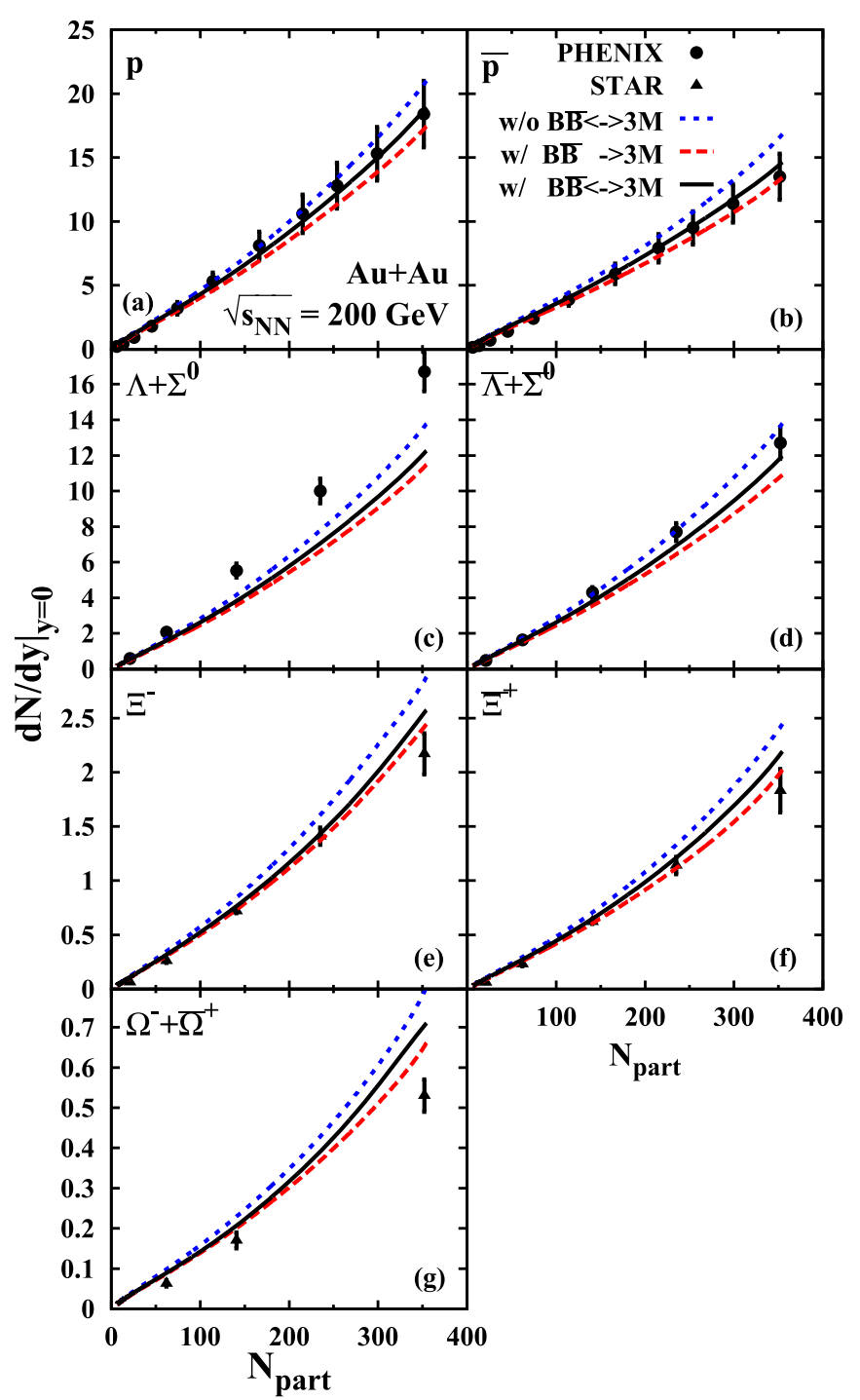

FIG. 7. The rapidity density of baryons and antibaryons from PHSD at midrapidity in comparison to data from the PHENIX and STAR Collaborations $[48,54,55]$ for $5 \%$ central $\mathrm{Au}+\mathrm{Au}$ collisions at $\sqrt{s_{N N}}=200 \mathrm{GeV}$. The dashed red lines show the results of calculations with only $B \bar{B}$ annihilation, the solid black lines show results with the $2 \leftrightarrow 3$ reactions included, while dotted blue lines correspond to calculations with the $2 \leftrightarrow 3$ reactions discarded.

channels wash out the memory from the initial production to a large extent.

\section{Centrality dependence of baryons and antibaryons at RHIC and LHC}

We continue with $p_{T}$ integrated rapidity densities for baryons and antibaryons as a function of centrality in terms of the number of participating nucleons $N_{\text {part }}$ which is calculated within PHSD. Figure 7 shows the rapidity density of baryons and antibaryons from PHSD at midrapidity in comparison to data from the PHENIX and STAR Collaborations $[48,54,55]$ for $5 \%$ central $\mathrm{Au}+\mathrm{Au}$ collisions at $\sqrt{s_{N N}}=200 \mathrm{GeV}$. When discarding the $2 \leftrightarrow 3$ reactions (blue dotted lines) the

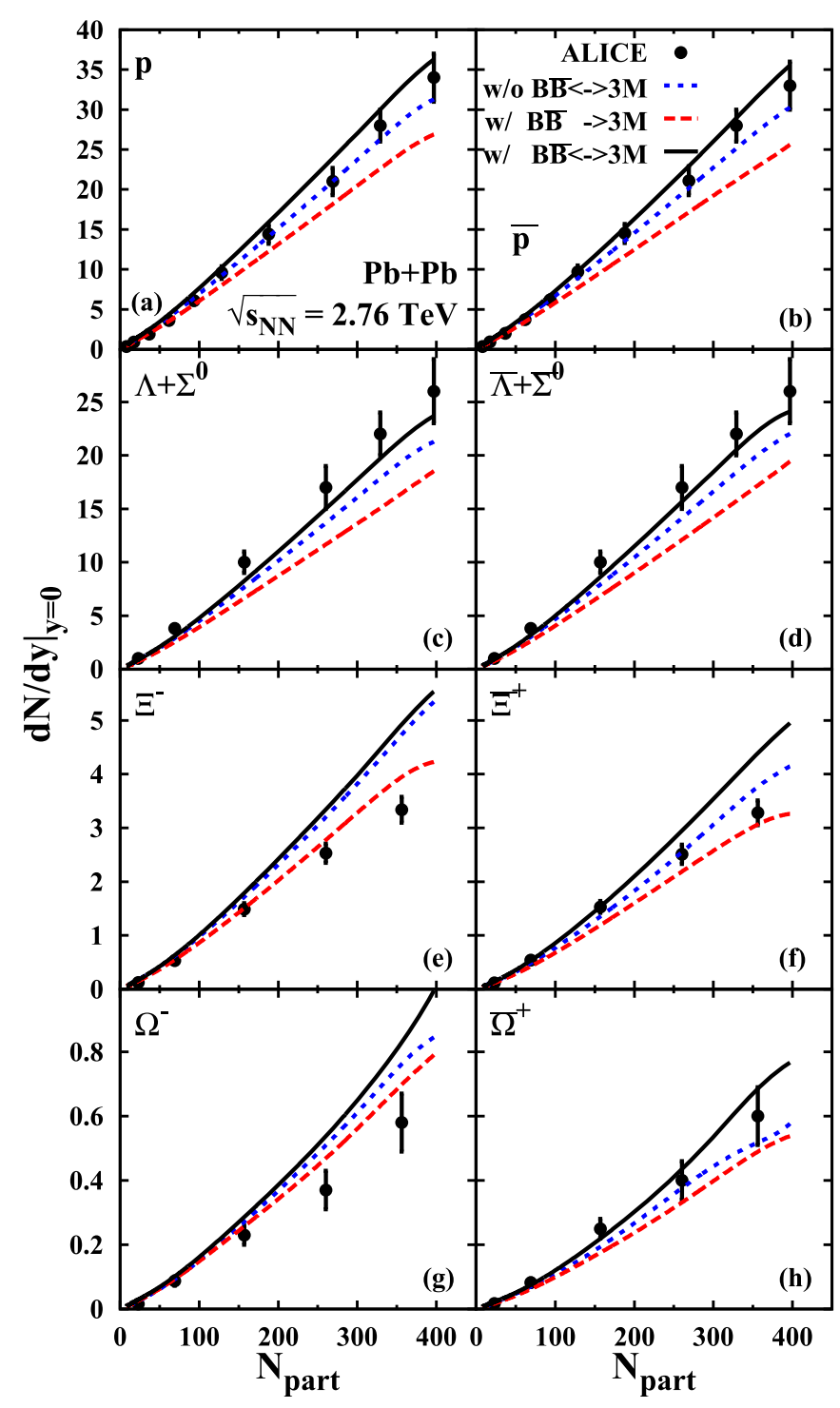

FIG. 8. The rapidity density of baryons and antibaryons from PHSD at midrapidity in comparison to the data from the ALICE Collaboration $[19,49-52]$ for $5 \%$ central $\mathrm{Pb}+\mathrm{Pb}$ collisions at $\sqrt{s_{N N}}=$ $2.76 \mathrm{TeV}$. The dashed red lines show the results of calculations with only $B \bar{B}$ annihilation, the solid black lines show results with the $2 \leftrightarrow 3$ reactions included, while dotted blue lines correspond to calculations with the $2 \leftrightarrow 3$ reactions discarded.

experimental data are slightly overestimated (except for $\Lambda+$ $\Sigma^{0}$ ), while calculations with only $B \bar{B}$ annihilation (dashed red lines) show a slight tendency to underestimate the data. The results from PHSD calculations with the $2 \leftrightarrow 3$ reactions included are displayed by the black solid lines and lie in between the other limits. This points towards a small net $B \bar{B}$ annihilation at the top RHIC energy for all baryons and antibaryons considered. We will quantify this net annihilation in Sec. IV.

The situation is somewhat different at LHC energies. Figure 8 shows the rapidity density of baryons and antibaryons from PHSD at midrapidity in comparison to data from the ALICE Collaboration [19,49-52] for $5 \%$ central $\mathrm{Pb}+\mathrm{Pb}$ 
collisions at $\sqrt{s_{N N}}=2.76 \mathrm{TeV}$. The blue dotted lines display the calculated results when discarding the $2 \leftrightarrow 3$ reactions and the dashed red lines correspond to calculations with only $B \bar{B}$ annihilation. The results from PHSD calculations with the $2 \leftrightarrow 3$ reactions included are displayed by the black solid lines and lie in all cases slightly above the other limits indicating a net $B \bar{B}$ production at the LHC instead of an absorption. The calculations with only $B \bar{B}$ annihilation (red dashed line) underestimate the experimental data (except for $\Xi^{-}$and $\Omega^{-}$). In particular the $p, \bar{p}, \Lambda+\Sigma^{0}$, and $\bar{\Lambda}+\bar{\Sigma}^{0}$ multiplicities are (within error bars) in line with experimental observation at all centralities (when including the $B \bar{B} \leftrightarrow 3 M$ channels) contrary to the results of the statistical hadronization model (SHM) quoted in Ref. [19]. On the other hand, the $\Xi^{-}, \bar{\Xi}^{+}, \Omega^{-}$, and $\bar{\Omega}^{+}$baryons are slightly overestimated in more central collisions when including the $B \bar{B} \leftrightarrow 3 M$ channels. We attribute these results to a deviation from statistical equilibrium in the hadronization incorporated in PHSD.

\section{EXCITATION FUNCTIONS}

In this section we will quantify the net effect of the $B \bar{B} \leftrightarrow$ $3 M$ channels for central $\mathrm{Pb}+\mathrm{Pb}(\mathrm{Au}+\mathrm{Au})$ collisions as a function of the bombarding energy or $\sqrt{s_{N N}}$, respectively, including the previous results from Ref. [31].

\section{A. Hadron yields at midrapidity}

In Fig. 9 we first show the performance of PHSD4.0 with respect to hadron production (at midrapidity) in central $\mathrm{Pb}+\mathrm{Pb}$ $(\mathrm{Au}+\mathrm{Au})$ collisions from $\sqrt{s_{N N}}=3.5 \mathrm{GeV}$ to $2.76 \mathrm{TeV}$, i.e., by roughly 3 orders of magnitude in invariant energy. The solid lines refer to calculations including the $B \bar{B} \leftrightarrow 3 M$ channels while the dashed lines display calculations without these channels. The particle yields at midrapidity from PHSD are connected by lines (to draw the eye) although experimental data (taken from Refs. [19,48-52,54-68]) and calculations do not always correspond to the same centrality selection (and system) for different bombarding energies. However, for given $\sqrt{s_{N N}}$, the data and calculation correspond to the same centrality and collision system. From Fig. 9(a) we see that PHSD essentially reproduces the experimental observations for pions, kaons, and antikaons in the whole energy range. We recall that at AGS and SPS energies this is essentially due to the incorporation of chiral symmetry restoration (cf. Refs. [35,36]). The same holds true for the baryon and antibaryon excitation functions except for the energy regime $20 \mathrm{GeV}<\sqrt{s_{N N}}<100 \mathrm{GeV}$ where PHSD underestimates the baryons and antibaryons. The reason for this discrepancy is presently not understood. However, by comparing the hadron yields from calculations with (solid lines) and without (dashed lines) the $B \bar{B} \leftrightarrow 3 M$ channels we find no essential differences by eye.

\section{B. Quantitative impact of many-body reactions}

In this subsection we will quantify the effect of the $B \bar{B} \leftrightarrow$ $3 M$ channels and $B \bar{B} \rightarrow 3 M$ channels in $5 \%$ central $\mathrm{Pb}+\mathrm{Pb}$ collisions for $3.5 \mathrm{GeV} \leqslant \sqrt{s_{N N}} \leqslant 2.76 \mathrm{TeV}$. To this end we show in Fig. 10 the ratio of the antibaryons $\bar{p}, \bar{\lambda}+\bar{\Sigma}^{0}, \bar{\Xi}$, and $\bar{\Omega}$ (at midrapidity) from PHSD calculations including the

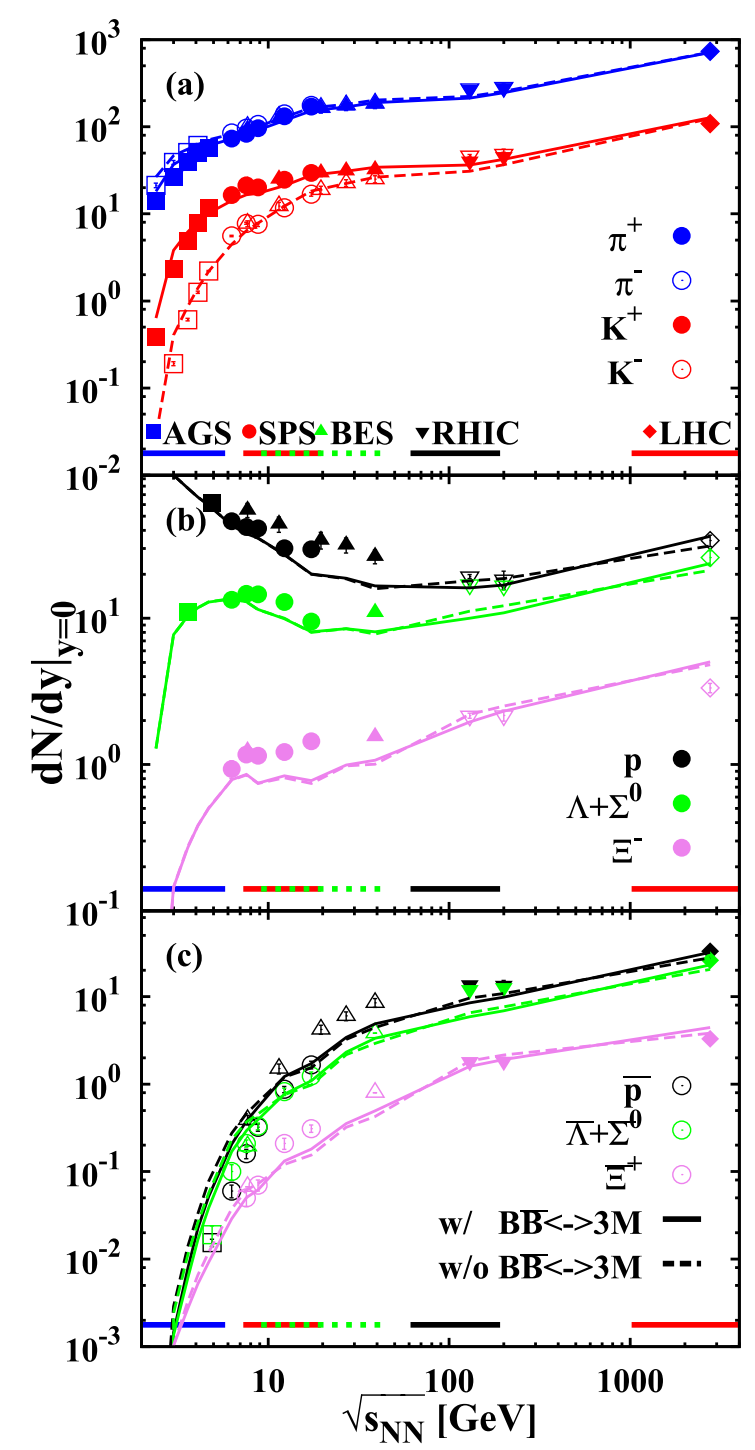

FIG. 9. The midrapidity yields of mesons (a), baryons (b), and antibaryons (c) from PHSD as a function of the invariant energy $\sqrt{s_{N N}}$ for central heavy-ion collisions in comparison with the experimental data taken from Refs. [19,48-52,54-68]. The solid lines refer to calculations including the $B \bar{B} \leftrightarrow 3 M$ channels while the dashed lines display calculations without these channels. The particle yields from PHSD are connected by lines to draw the eye although experimental data and calculations do not always correspond to the same centrality selection (and system) for different bombarding energies.

$B \bar{B} \leftrightarrow 3 M$ channels to calculations without them. At low $\sqrt{s_{N N}} \approx 3.5 \mathrm{GeV}$ we observe a sizable net annihilation of antiprotons and antihyperons by about a factor of two which is essentially due to the fact that here the nucleon density is very large compared to the antinucleon density. Practically the same holds for the strangeness $S= \pm 1$ sector while the net suppression of $\bar{\Xi}^{+}$is only $20 \%$. For $\bar{\Omega}^{+}$'s there is no net suppression within error bars which results from the statistical errors of both calculations. With increasing invariant energy the net annihilation of antiprotons and antihyperons disappears at $\sqrt{s_{N N}} \approx 10 \mathrm{GeV}$, i.e., at the top SPS and lower RHIC energies. For $\sqrt{s_{N N}}=130 \mathrm{GeV}$ and $200 \mathrm{GeV}$ we find a small 


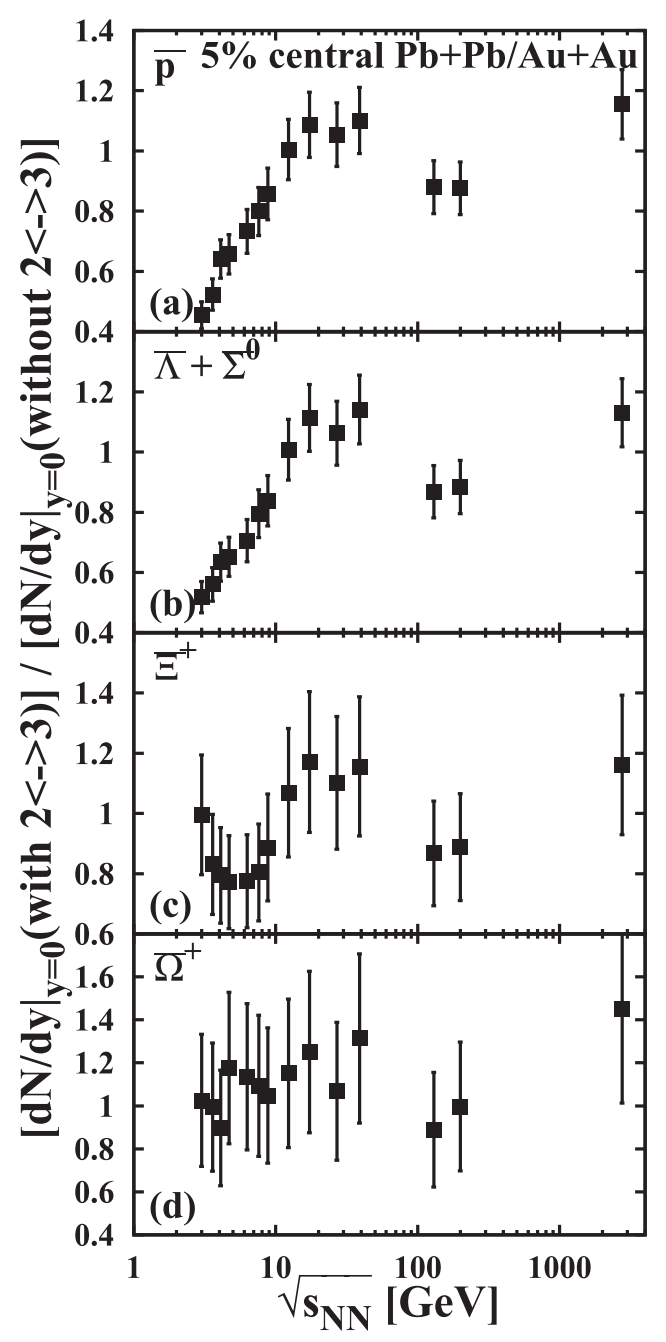

FIG. 10. Ratios of $0-5 \%$ central midrapidity yields from calculations with the full $B \bar{B} \leftrightarrow 3 M$ reactions to calculations without them for the antibaryons as a function of the invariant energy $\sqrt{s_{N N}}$.

net annihilation for $\bar{p}, \bar{\Lambda}+\bar{\Sigma}^{0}$, and $\bar{\Xi}^{+}$which turns to a small enhancement at the LHC energy of $\sqrt{s_{N N}}=2.76 \mathrm{TeV}$ as noted before. This is in contrast to the results of the model calculations in Refs. $[20,25]$. The small net suppression of antiprotons at the top RHIC energy, however, is in line with the results from Ref. [26] which also incorporate detailed balance for the annihilation channels. We interpret the tiny enhancement of antibaryons at the LHC energy to result from the huge meson abundances which in phase space are slightly overpopulated in PHSD relative to baryon-antibaryon pairs at hadronization.

In order to investigate the effect of the $B \bar{B}$ annihilation channels we show in Fig. 11 the ratio of the antibaryons $\bar{p}, \bar{\Lambda}+$ $\bar{\Sigma}^{0}, \bar{\Xi}^{+}$, and $\bar{\Omega}^{+}$(at midrapidity) from PHSD calculations including the $B \bar{B} \leftrightarrow 3 M$ channels to calculations with only the annihilation channels for the same reactions as in Fig. 10. Although this ratio is an unphysical quantity, it allows us to shed light on the relative importance of the annihilation channels. For all antibaryons in Fig. 11 this ratio is larger than unity, which implies that the back-reactions have some

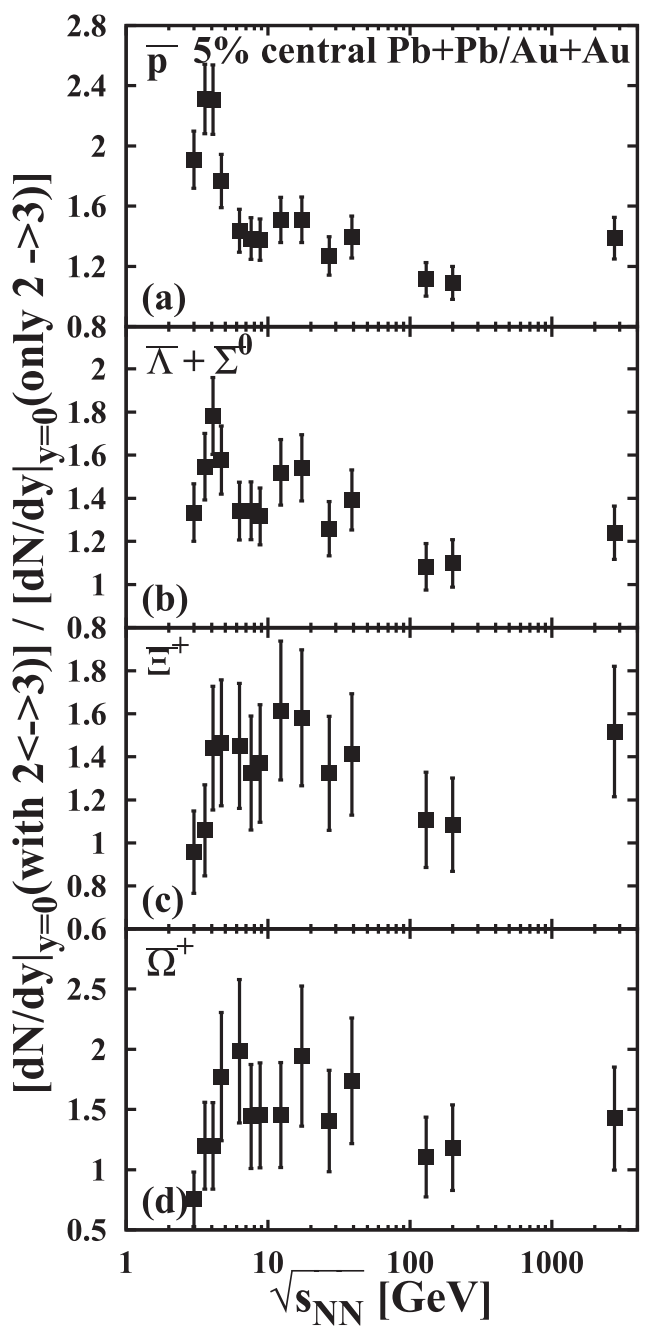

FIG. 11. Ratios of 0-5\% central midrapidity yields from PHSD calculations with the full $B \bar{B} \leftrightarrow 3 M$ reactions to calculations with only annihilation as a function of the invariant energy $\sqrt{s_{N N}}$.

impact on the final antibaryon multiplicities. This effect is most pronounced at lower SPS energies, where the baryon densities are large compared to the antibaryon densities and drops below $50 \%$ enhancement for invariant energies above about $\sqrt{s_{N N}}=10 \mathrm{GeV}$ (within error bars). At top RHIC and LHC energies these modifications are below the $20 \%$ level since baryon and antibaryon densities are comparable and all elastic and inelastic $2 \leftrightarrow 2$ channels are equal for time reversed states. Only the relative weight of baryons to mesons changes slightly resulting in ratios greater than unity.

\section{SUMMARY}

In this study we have employed the extended quark rearrangement model for baryon-antibaryon annihilation $(B \bar{B} \leftrightarrow$ $3 M$ ) from Ref. [31] - incorporated in PHSD4.0 - for the hadron production in heavy-ion collisions at ultrarelativistic energies. We recall-using simulations in a box with periodic boundary conditions - that the numerical implementation of the quark rearrangement model including the strangeness 


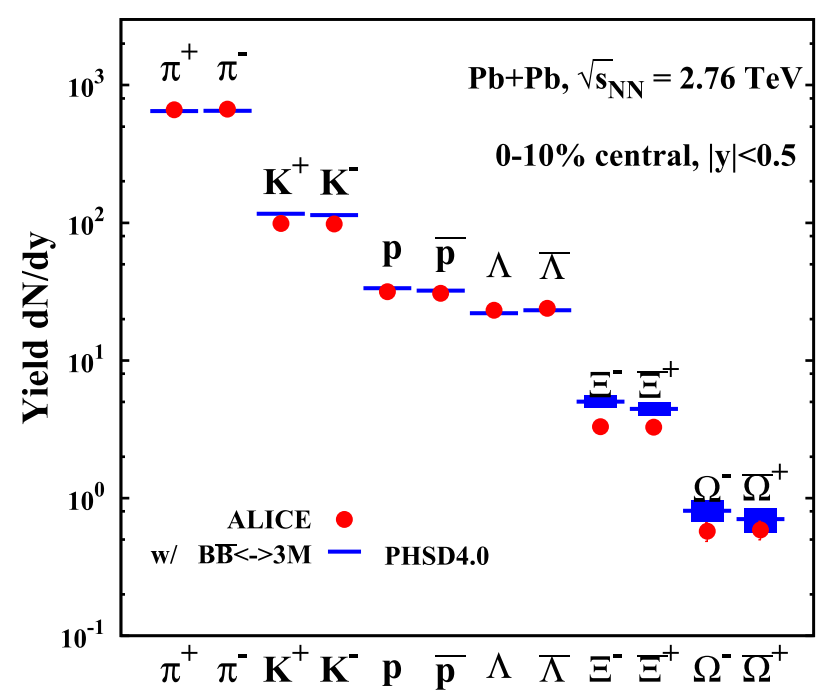

FIG. 12. The midrapidity yields from PHSD4.0 including the $B \bar{B} \leftrightarrow 3 M$ reactions (solid lines) for $0-10 \%$ central $\mathrm{Pb}+\mathrm{Pb}$ collisions at $\sqrt{s_{N N}}=2.76 \mathrm{TeV}$. The data points are taken from Ref. [18].

sector satisfies the detailed balance $2 \leftrightarrow 3$ relations on a channel-by-channel basis as well as differentially as a function of the invariant energy $\sqrt{s}$ [31]. It has been found that the effects from the $(B \bar{B} \leftrightarrow 3 M)$ reaction channels on the meson, baryon, and antibaryon spectra is only moderate, although nonzero. At the top RHIC energy we find a small net suppression of $B \bar{B}$ pairs relative to calculations without these channels, whereas at the LHC energy of $\sqrt{s_{N N}}=2.76 \mathrm{TeV}$ there is even a net enhancement of $B \bar{B}$ pairs which we attribute to the higher meson densities. The PHSD net antibaryon enhancement is in contrast to the results of the model calculations in Refs. [20,25] at the LHC energy, whereas the small net suppression of antiprotons at the top RHIC energy is in line with the results from Ref. [26] which also incorporate detailed balance for the annihilation channels. Accordingly, the sizable difference between data and statistical calculations in $\mathrm{Pb}+\mathrm{Pb}$ collisions at $\sqrt{s_{N N}}=2.76 \mathrm{TeV}$ for proton and antiproton yields [19], where a deviation of $2.7 \sigma$ was obtained [18], should not be attributed to a net antiproton annihilation. On the other hand, no substantial deviation between the data and SHM calculations is seen for antihyperons [21], which, according to the PHSD analysis, should be modified by the same amount as antiprotons (cf. Fig. 10).

To summarize our results for the LHC energy we show in Fig. 12 the particle ratios at midrapidity from $10 \%$ central $\mathrm{Pb}+\mathrm{Pb}$ collisions in comparison to the data from the ALICE Collaboration. Since the PHSD results are in line with data (within error bars) this points towards a possible deviation from statistical equilibrium in the hadronization (at least for protons and antiprotons).

Furthermore, we find that the $B \bar{B} \leftrightarrow 3 M$ reactions are more important at lower SPS or Facility for Antiproton and Ion Research and the Nuclotron-Based Ion Collider Facility (FAIR and NICA) energies where a net suppression for antiprotons and antihyperons up to a factor of $2-2.5$ is seen in the PHSD calculations (cf. Fig. 10). In this energy regime further data on antibaryons (also with multiple strangeness) will be needed with high statistics as a function of centrality and system size; a task well suited for the upcoming facilities at FAIR and NICA. So far the baryon-antibaryon dynamics is not sufficiently understood and open for further analysis.

\section{ACKNOWLEDGMENTS}

The authors acknowledge inspiring discussions with E. L. Bratkovskaya, P. Moreau, A. Palmese, T. Steinert, and R. Stock. We thank the Helmholtz International Center for FAIR (HIC for FAIR), the Helmholtz Graduate School for Hadron and Ion Research (HGS-HIRe), and the Helmholtz Research School for Quark Matter Studies in Heavy-Ion Collisions (H-QM) for support. The computational resources have been provided by the Center for Scientific Computing (CSC) in the framework of the Landes-Offensive zur Entwicklung Wissenschaftlich-ökonomischer Exzellenz (LOEWE) and the Green IT Cube at FAIR.
[1] Y. Aoki et al., Phys. Lett. B 643, 46 (2006).

[2] S. Borsanyi et al., J. High Energy Phys. 09 (2010) 073; 11 (2010) 077; 08 (2012) 126.

[3] S. Borsanyi et al., Phys. Lett. B 730, 99 (2014); Phys. Rev. D 92, 014505 (2015).

[4] P. Petreczky (HotQCD Collaboration), PoS LATTICE 2012, 069 (2012); AIP Conf. Proc. 1520, 103 (2013).

[5] H.-T. Ding, F. Karsch, and S. Mukherjee, Int. J. Mod. Phys. E 24, 1530007 (2015).

[6] A. Bazavov, T. Bhattacharya, C. DeTar, H.T. Ding, S. Gottlieb, R. Gupta, P. Hegde, U.M. Heller, F. Karsch, E. Laermann, L. Levkova, S. Mukherjee, P. Petreczky, C. Schmidt, C. Schroeder, R.A. Soltz, W. Soeldner, R. Sugar, M. Wagner, and P. Vranas, Phys. Rev. D 90, 094503 (2014).

[7] P. Senger et al., Lect. Notes Phys. 814, 681 (2011).

[8] A. Bazavov, H. T. Ding, P. Hegde, O. Kaczmarek, F. Karsch, E. Laermann, Y. Maezawa, S. Mukherjee, H. Ohno, P. Petreczky,
H. Sandmeyer, P. Steinbrecher, C. Schmidt, S. Sharma, W. Soeldner, and M. Wagner, Phys. Rev. D 95, 054504 (2017).

[9] F. Karsch, PoS CPOD2013, 046 (2013).

[10] F. Becattini and R. Fries, Landolt-Bornstein 23, 208 (2010).

[11] Braun-Munzinger, K. Redlich, and J. Stachel, in Quark-Gluon Plasma 3, edited by H. C. Hwa and X. N. Wang (World Scientific, Singapore, 2004), p. 491.

[12] F. Becattini, M. Gaździcki, A. Keränen, J. Manninen, and R. Stock, Phys. Rev. C 69, 024905 (2004).

[13] A. Andronic et al., Nucl. Phys. A 772, 167 (2006).

[14] J. Rafelski and M. Danos, Phys. Lett. B 97, 167 (1980).

[15] A. Tounsi and K. Redlich, J. Phys. G 28, 2095 (2002).

[16] P. Braun-Munzinger, V. Koch, T. Schäfer, and J. Stachel, Phys. Rept. 621, 76 (2016).

[17] A. Andronic, D. Blaschke, P. Braun-Munzinger, J. Cleymans, K. Fukushima et al., Nucl. Phys. A 837, 65 (2010). 
[18] A. Andronic, P. Braun-Munzinger, K. Redlich, and J. Stachel, arXiv:1710.09425.

[19] B. Abelev et al. (ALICE Collaboration), Phys. Rev. C 88, 044910 (2013).

[20] F. Becattini, M. Bleicher, T. Kollegger, M. Mitrovski, T. Schuster, and R. Stock, Phys. Rev. C 85, 044921 (2012); F. Becattini et al., Phys. Rev. Lett. 111, 082302 (2013); F. Becattini et al., Phys. Lett. B 764, 241 (2017).

[21] J. Adam et al. (ALICE Collaboration), Nat. Phys. 13, 535 (2017).

[22] S. A. Bass et al., Prog. Part. Nucl. Phys. 41, 255 (1998).

[23] M. Bleicher et al., J. Phys. G 25, 1859 (1999).

[24] F. Becattini, M. Bleicher, J. Steinheimer, and R. Stock, arXiv:1712.03748.

[25] Y. Pan and S. Pratt, Phys. Rev. C 89, 044911 (2014).

[26] P. Huovinen and J. I. Kapusta, Phys. Rev. C 69, 014902 (2004).

[27] W. Cassing, Nucl. Phys. A 700, 618 (2002).

[28] W. Cassing and E. L. Bratkovskaya, Phys. Rep. 308, 65 (1999).

[29] H. Weber, E. L. Bratkovskaya, W. Cassing, and H. Stöcker, Phys. Rev. C 67, 014904 (2003).

[30] E. L. Bratkovskaya, M. Bleicher, M. Reiter, S. Soff, H. Stöcker, M. van Leeuwen, S. A. Bass, and W. Cassing, Phys. Rev. C 69, 054907 (2004).

[31] E. Seifert and W. Cassing, Phys. Rev. C 97, 024913 (2018).

[32] W. Cassing and E. L. Bratkovskaya, Phys. Rev. C 78, 034919 (2008).

[33] W. Cassing and E. Bratkovskaya, Nucl. Phys. A 831, 215 (2009).

[34] E. L. Bratkovskaya, W. Cassing, V. P. Konchakovski, and O. Linnyk, Nucl. Phys. A 856, 162 (2011).

[35] W. Cassing, A. Palmese, P. Moreau, and E. L. Bratkovskaya, Phys. Rev. C 93, 014902 (2016).

[36] A. Palmese, W. Cassing, E. Seifert, T. Steinert, P. Moreau, and E. L. Bratkovskaya, Phys. Rev. C 94, 044912 (2016).

[37] O. Linnyk, E. L. Bratkovskaya, and W. Cassing, Prog. Part. Nucl. Phys. 87, 50 (2016).

[38] O. Linnyk, V. P. Konchakovski, W. Cassing, and E. L. Bratkovskaya, Phys. Rev. C 88, 034904 (2013); O. Linnyk et al., ibid. 92, 054914 (2015).

[39] V. P. Konchakovski, E. L. Bratkovskaya, W. Cassing, V. D. Toneev, S. A. Voloshin, and V. Voronyuk, Phys. Rev. C 85, 044922 (2012).

[40] L. P. Kadanoff and G. Baym, Quantum Statistical Mechanics (Benjamin, New York, 1962).

[41] J. S. Schwinger, J. Math. Phys. 2, 407 (1961).

[42] L. V. Keldysh, Zh. Eksp. Teor. Fiz. 47, 1515 (1964) [Sov. Phys. JETP 20, 1018 (1965)].

[43] W. Botermans and R. Malfliet, Phys. Rept. 198, 115 (1990).

[44] W. Cassing and S. Juchem, Nucl. Phys. A 665, 377 (2000); 672, 417 (2000).

[45] A. Peshier, Phys. Rev. D 70, 034016 (2004).

[46] A. Peshier, J. Phys. G 31, S371 (2005).

[47] B. Nilsson-Almqvist and E. Stenlund, Comput. Phys. Commun. 43, 387 (1987).
[48] S. S. Adler et al. (PHENIX Collaboration), Phys. Rev. C 69, 034909 (2004).

[49] K. Aamodt et al. (ALICE Collaboration), Phys. Rev. Lett. 106, 032301 (2011).

[50] B. Abelev et al. (ALICE Collaboration), Phys. Lett. B 720, 52 (2013); Phys. Rev. Lett. 111, 222301 (2013); Phys. Lett. B 728, 216 (2014).

[51] E. Abbas et al. (ALICE Collaboration), Phys. Lett. B 726, 610 (2013); Phys. Rev. Lett. 111, 162301 (2013).

[52] J. Adam et al. (ALICE Collaboration), Phys. Lett. B 754, 373 (2016).

[53] V. P. Konchakovski, W. Cassing, and V. D. Toneev, J. Phys. G 42, 055106 (2015).

[54] G. Agakishiev et al. (STAR Collaboration), Phys. Rev. Lett. 108, 072301 (2012).

[55] J. Adams et al. (STAR Collaboration), Phys. Rev. Lett. 98, 062301 (2007).

[56] K. Adcox et al. (PHENIX Collaboration), Phys. Rev. Lett. 89, 092302 (2002); Phys. Rev. C 69, 024904 (2004).

[57] C. Adler et al. (STAR Collaboration), Phys. Rev. Lett. 89, 092301 (2002).

[58] J. Adams et al. (STAR Collaboration), Phys. Rev. Lett. 92, 182301 (2004).

[59] L. Adamczyk et al. (STAR Collaboration), Phys. Rev. C 96, 044904 (2017).

[60] L. Ahle et al. (E802 Collaboration), Phys. Rev. Lett. 81, 2650 (1998).

[61] L. Ahle et al. (E866 and E917 Collaborations), Phys. Lett. B 476, 1 (2000); 490, 53 (2000).

[62] B. B. Back, R. R. Betts, J. Chang, W. C. Chang, C. Y. Chi, Y. Y. Chu, J. B. Cumming, J. C. Dunlop, W. Eldredge, S. Y. Fung, R. Ganz, E. Garcia, A. Gillitzer, G. Heintzelman, W. F. Henning, D. J. Hofman, B. Holzman, J. H. Kang, E. J. Kim, S. Y. Kim, Y. Kwon, D. McLeod, A. C. Mignerey, M. Moulson, V. Nanal, C. A. Ogilvie, R. Pak, A. Ruangma, D. E. Russ, R. Seto, P. J. Stanskas, G. S. F. Stephans, H. Wang, F. L. H. Wolfs, A. H. Wuosmaa, H. Xiang, G. H. Xu, H. B. Yau, and C. M. Zou (E917 Collaboration), Phys. Rev. Lett. 87, 242301 (2001).

[63] J. L. Klay et al. (E-0895 Collaboration), Phys. Rev. C 68, 054905 (2003).

[64] P. Chung et al. (E895 Collaboration), Phys. Rev. Lett. 91, 202301 (2003).

[65] S. V. Afanasiev et al. (NA49 Collaboration), Phys. Rev. C 66, 054902 (2002).

[66] C. Alt et al. (NA49 Collaboration), Phys. Rev. Lett. 94, 192301 (2005); Phys. Rev. C 73, 044910 (2006); 77, 024903 (2008); 78, 034918 (2008).

[67] T. Anticic et al. (NA49 Collaboration), Phys. Rev. Lett. 93, 022302 (2004); Phys. Rev. C 80, 034906 (2009); 83, 014901 (2011).

[68] M. K. Mitrovski et al. (NA49 Collaboration), J. Phys. G 32, S43 (2006). 